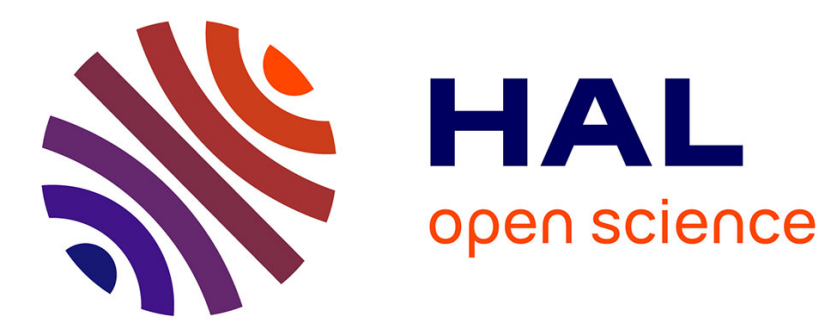

\title{
Extension of SmEdA (Statistical model Energy distribution Analysis) to non-resonant transmission
}

Laurent Maxit, Kerem Ege, Nicolas Totaro, Jean-Louis Guyader

\section{To cite this version:}

Laurent Maxit, Kerem Ege, Nicolas Totaro, Jean-Louis Guyader. Extension of SmEdA (Statistical model Energy distribution Analysis) to non-resonant transmission. 21st International Congress on Acoustics (ICA 2013), Jun 2013, Montréal, Canada. pp.065076, 10.1121/1.4799564 . hal-00823977

\section{HAL Id: hal-00823977 \\ https://hal.science/hal-00823977}

Submitted on 19 May 2016

HAL is a multi-disciplinary open access archive for the deposit and dissemination of scientific research documents, whether they are published or not. The documents may come from teaching and research institutions in France or abroad, or from public or private research centers.
L'archive ouverte pluridisciplinaire HAL, est destinée au dépôt et à la diffusion de documents scientifiques de niveau recherche, publiés ou non, émanant des établissements d'enseignement et de recherche français ou étrangers, des laboratoires publics ou privés. 
Extension of SmEdA (Statistical model Energy distribution Analysis) to nonresonant transmission

Laurent Maxit, Kerem Ege, Nicolas Totaro and Jean-Louis Guyader

Laboratoire Vibrations Acoustique, INSA-Lyon, 25 bis Avenue Jean Capelle,

F-69621 Villeurbanne Cedex, France 


\section{INTRODUCTION}

Statistical Energy Analysis was used in the past for evaluating the sound transmission of structures: simple/double panels separating two rooms [1,2], timber floor [3] or of a hybrid heavyweight-lightweight floor [4]. A limitation of this approach is that it cannot be easily applied to evaluate the sound transmission through nonhomogenous or stiffened panels like the firewall of an automotive or the compartment partitions of a rail vehicle. Moreover, this method is not adapted to evaluate the influence on transmission loss of excitation conditions such as source room geometry, source location, or the location of the panel in the source room [5]. On another hand, as the non resonant transmission is not described in the classical SEA model which describes the energy sharing between the resonant modes, Crocker and Price [1] introduced an indirect coupling loss factor between the excited and the receiving room. The parameter is estimated from the simple mass law equation of an infinite panel for frequencies below the critical frequency. In [6], Craik proposes a new formula of this coupling loss factor when dealing with a double wall. It seems that this parameter depends on the considered structure.

In this paper, one proposes to evaluate the sound transmission through a complex panel separating two cavities by using the Statistical model Energy distribution Analysis (SmEdA) model [7, 8]. This method is based on the same assumptions as the Statistical Energy Analysis (SEA) model except for the modal energy equipartition assumption which is not supposed by the SmEdA model. This method has the advantage of using the modal bases of uncoupled subsystems. These bases can be evaluated by using Finite Element models when subsystems have a complex geometry ([9]). As SEA, SmEdA describes the energy sharing by the resonant modes. One extends here the SmEdA model for describing the contribution of the non resonant modes.

\section{DESCRIPTION OF CAVITY-STRUCTURE-CAVITY SYSTEM WITH THE DUAL MODAL FORMULATION}

Let us consider the cavity-structure-cavity problem represented on Fig. 1. It is composed of two air acoustic cavities and an elastic thin structure. The two cavities can exchange vibrating energy though the thin structure and all the others cavity walls are supposed as rigid. One supposes that an acoustic source is located into the cavity 1 and has a white noise spectrum in the frequency band $\left[\tilde{\omega}_{1}, \tilde{\omega}_{2}\right]$. Then, by estimating the total energy of each cavity, one could deduce the transmission loss of the test structure.

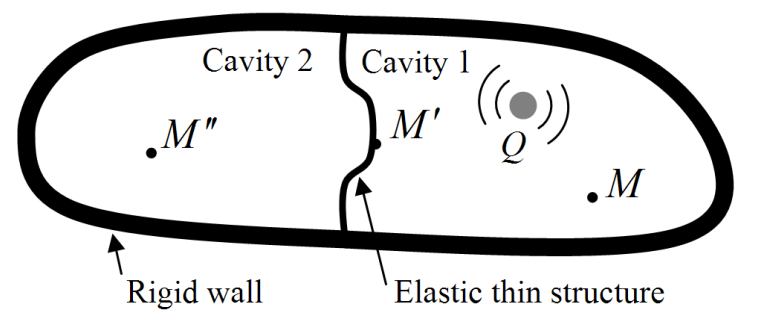

FIGURE 1. Illustration of the cavity-structure-cavity system considered to evaluate the sound transmission of a test structure.

This vibro-acoustic problem can be described by using the Dual Modal Formulation (DMF) [8, 10]. In accordance with this formulation, the thin structure is described by a displacement and its uncoupled-free modes whereas the cavities are described by a stress field and their uncoupled-blocked modes. The boundary conditions of these uncoupled subsystem modes are illustrated on Fig. 2 for the present case. These subsystem modes can be easily calculated analytically for academic cases ([10]) or numerically with Finite Elements models for complex cases ([9]). 


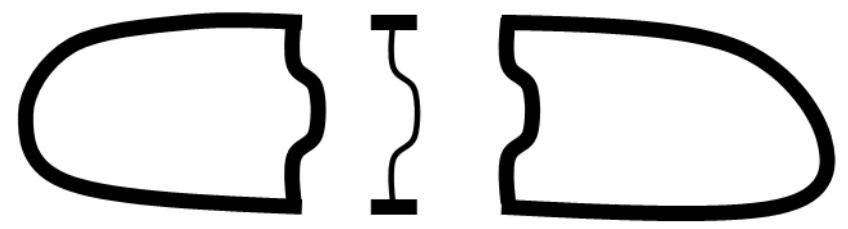

FIGURE 2. Illustration of the uncoupled subsystems with appropriate boundary conditions.

The modal expansions of the normal displacement $W$ at point $M^{\prime}$ on the thin structure may be written

$$
W\left(M^{\prime}, t\right)=\sum_{q \in \hat{Q}} \chi_{q}(t) \tilde{W}_{q}\left(M^{\prime}\right),
$$

whereas the acoustic pressures $p$ at point $M$ and $M^{\prime \prime}$ inside cavity 1 and 2, respectively, write:

$$
p(M, t)=\sum_{p \in \hat{P}} \varphi_{p}^{\prime}(t) \tilde{p}_{p}(M), p\left(M^{\prime \prime}, t\right)=\sum_{r \in \hat{R}} \zeta^{\prime}(t) \tilde{p}_{r}\left(M^{\prime \prime}\right),
$$

where:

$-\phi_{p}, \chi_{q}, \xi_{r}$ are the modal amplitudes;

- $\tilde{p}_{p}, \tilde{p}_{r}$ are the pressure mode shape (normalised to a unit modal stiffness) of cavity 1 and 2 , respectively;

- $\tilde{W}_{q}$ are the displacement mode shapes (normalised to a unit modal mass) of the thin structure.

The modal summations of these equations should theoretically have an infinite number of terms. For practical reason, it is necessary to truncate them. Let us consider a finite set of modes for each subsystem. One note $\hat{P}, \hat{Q}$, and $\hat{R}$ the mode sets, respectively, for the cavity 1 , the structure and the cavity 2 .

DMF consists in introducing these expansions (1-2) in a weak formulation of the considered vibro-acoustic problem and using the orthogonality properties of the uncoupled modes. For more details on this formulation, the reader can study Ref. [8]. The following modal equations are obtained

$$
\left\{\begin{array}{l}
\varphi_{p}^{\prime \prime}+\omega_{p} \eta_{p} \varphi_{p}^{\prime}+\omega_{p}^{2} \varphi_{p}-\sum_{q \in \hat{Q}} W_{p q} \chi_{q}^{\prime}=Q_{p}, \forall p \in \hat{P} \\
\chi_{q}^{\prime \prime}+\omega_{q} \eta_{q} \chi_{q}^{\prime}+\omega_{q}^{2} \chi_{q}+\sum_{p \in \hat{P}} W_{p q} \varphi_{p}^{\prime}+\sum_{r \in \hat{R}} W_{r q} \zeta_{r}^{\prime}=0, \forall q \in \hat{Q} \\
\zeta_{r}^{\prime \prime}+\omega_{r} \eta_{r} \zeta_{r}^{\prime}+\omega_{r}^{2} \zeta_{r}-\sum_{q \in \hat{Q}} W_{r q} \chi_{q}^{\prime}=0, \forall r \in \hat{R}
\end{array}\right.
$$

where:

- $Q_{p}$ are the generalised source strength due to the acoustic source $Q_{0}$;

$-\omega_{p}, \omega_{q}, \omega_{r}$ are the angular frequencies of subsystem modes;

- $\eta_{p}, \eta_{q}, \eta_{r}$ are the modal damping loss factors, and ;

- $W_{p q}, W_{q r}$ are the modal interaction works defined by:

$$
W_{p q}=\int_{S} \tilde{W}_{q} \tilde{p}_{p} d S, W_{q r}=\int_{S} \tilde{W}_{q} \tilde{p}_{r} d S .
$$

The form of these equations allows us to interpret modes interactions as oscillators with gyroscopic couplings. Note that a mode of one subsystem is coupled to the modes of the other subsystem but is not directly coupled with the other modes of the subsystem to which it belongs. This configuration of mode coupling is exactly the one that assumes SEA. 


\section{SMEDA FORMULATION INCLUDING NON RESONANT TRANSMISSION}

In this section, we are interested by establishing an energy formulation of the vibro-acoustic behaviour of the cavity-structure-cavity system. The original SmEdA model [7] is based on the DMF described previously and allows us describing the energy sharing between the resonant modes (i.e. the modes contained in the frequency band of excitation $\left.\left[\tilde{\omega}_{1}, \tilde{\omega}_{2}\right]\right)$. For the case considered in this paper, it is well know that the Non-Resonant (NR) structure modes play an important role for frequencies below the critical frequency. Indeed, the low frequency structure modes which are not in frequency coincidence with the resonant cavity modes may be in space coincidence with these cavity modes and may participate significantly to the energy transmission between the two cavities. An illustration of the modal interaction for frequencies below the critical frequency is given in Fig. 3. The original SmEdA model describes only the resonant transmissions plotted in black on this figure. One extends here the SmEdA model for describing the energy exchanged by the low frequency NR structure modes (plotted in red on Fig. $3)$.

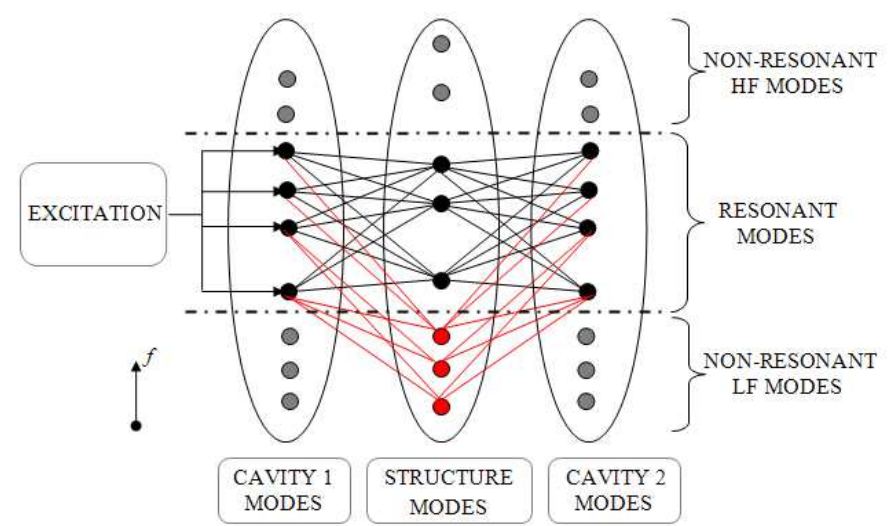

FIGURE 3. Modal interaction scheme of the sound transmission below the critical frequency.

\section{Non-Resonant Modes Condensation}

A new interpretation of the modal coupling shown in Fig. 3 is proposed in this section. Let us applying a Fourier transform to (3). Then, one can write the matrix system:

$$
\left[\begin{array}{ccc}
Z_{11} & -j \omega W_{12} & 0 \\
+j \omega W_{12}^{*} & Z_{22} & +j \omega W_{23}^{*} \\
0 & -j \omega W_{23} & Z_{33}
\end{array}\right]\left[\begin{array}{c}
\Gamma_{1} \\
\Gamma_{2} \\
\Gamma_{3}
\end{array}\right]=\left[\begin{array}{c}
Q_{1} \\
0 \\
0
\end{array}\right]
$$

with the modal amplitude and generalised source strength vectors:

$$
\Gamma_{1}=\left[\begin{array}{c}
\vdots \\
\bar{\varphi}_{p} \\
\vdots
\end{array}\right]_{P \times 1}, \Gamma_{2}=\left[\begin{array}{c}
\vdots \\
\bar{\chi}_{q} \\
\vdots
\end{array}\right]_{Q \times 1}, \Gamma_{3}=\left[\begin{array}{c}
\vdots \\
\bar{\zeta}_{r} \\
\vdots
\end{array}\right]_{R \times 1}, Q_{1}=\left[\begin{array}{c}
\vdots \\
\bar{Q}_{p} \\
\vdots
\end{array}\right]_{P \times 1},
$$

the modal impedance matrices and the modal interaction works,

$$
\begin{aligned}
& Z_{11}=\operatorname{diag}\left[-\omega^{2}+j \omega \omega_{p} \eta_{p}+\omega_{p}^{2}\right]_{P \times P}, Z_{22}=\operatorname{diag}\left[-\omega^{2}+j \omega \omega_{q} \eta_{q}+\omega_{q}^{2}\right]_{Q \times Q} \\
& Z_{33}=\operatorname{diag}\left[-\omega^{2}+j \omega \omega_{r} \eta_{r}+\omega_{r}^{2}\right]_{R \times R}, \text { and, } W_{12}=\left\lfloor W_{p q}\right\rfloor_{P \times Q}, W_{23}=\left[W_{q r}\right]_{Q \times R} .
\end{aligned}
$$

Now, let us considering two sets of modes for the structure: the resonant modes set, $\hat{Q}^{R}$ and the non resonant modes set, $\hat{Q}^{N R}$ which are defined by:

$$
q \in \hat{Q}^{N R} \Leftrightarrow \omega_{q} \in\left[0, \tilde{\omega}_{1}\left[\text { and } q \in \hat{Q}^{R} \Leftrightarrow \omega_{q} \in\left[\tilde{\omega}_{1}, \tilde{\omega}_{2}\right]\right.\right.
$$


The DMF equations in the frequency domain (5) can be rewritten:

$$
\left[\begin{array}{cccc}
Z_{11} & -j \omega W_{12}^{N R} & -j \omega W_{12}^{R} & 0 \\
+j \omega W_{12}^{N R^{*}} & Z_{22}^{N R} & 0 & +j \omega W_{23}^{N R^{*}} \\
+j \omega W_{12}^{R^{*}} & 0 & Z_{22}^{R} & +j \omega W_{23}^{R^{*}} \\
0 & -j \omega W_{23}^{N R} & -j \omega W_{23}^{R} & Z_{33}
\end{array}\right]\left[\begin{array}{c}
\Gamma_{1} \\
\Gamma_{2}^{N R} \\
\Gamma_{2}^{R} \\
\Gamma_{3}
\end{array}\right]=\left[\begin{array}{c}
Q_{1} \\
0 \\
0 \\
0
\end{array}\right]
$$

where the subscript R or NR are related to the Resonant and Non-Resonant modes sets respectively.

Eliminating $\Gamma_{2}^{N R}$ from the second row which can be reintroduced in the other rows gives the condensed matrix system:

$$
\left[\begin{array}{ccc}
Z_{11}-\omega^{2} W_{12}^{N R}\left[Z_{22}^{N R}\right]^{-1} W_{12}^{N R^{*}} & -j \omega W_{12}^{R} & -\omega^{2} W_{12}^{N R}\left[Z_{22}^{N R}\right]^{-1} W_{23}^{N R^{*}} \\
+j \omega W_{12}^{R} & Z_{22} & +j \omega W_{23}^{R} \\
-\omega^{2} W_{23}^{N R}\left[Z_{22}^{N R}\right]^{-1} W_{12}^{N R^{*}} & -j \omega W_{23}^{R} & Z_{33}-\omega^{2} W_{23}^{N R}\left[Z_{22}^{N R}\right]^{-1} W_{23}^{N R^{*}}
\end{array}\right]\left[\begin{array}{c}
\Gamma_{1} \\
\Gamma_{2}^{R} \\
\Gamma_{3}
\end{array}\right]=\left[\begin{array}{c}
Q_{1} \\
0 \\
0
\end{array}\right]
$$

This operation links the amplitudes of the resonant modes of the 3 subsystems. On another hand, if one considers that the natural frequencies of the NR modes are much lower than the angular frequency (i.e. $\omega_{q}<<\tilde{\omega}_{1}<\omega, \forall q \in \hat{Q}^{N R}$ ), one can assume: $\left[Z_{22}^{N R}\right]^{-1} \approx-\frac{1}{\omega^{2}} I$, where $I$ is the identity matrix $Q^{N R} \times Q^{N R}$. Moreover, if one neglects the matrix terms in addition to the modal impedance matrices of the cavities, one obtains:

$$
\left[\begin{array}{ccc}
Z_{11} & -j \omega W_{12}^{R} & -W_{12}^{N R} W_{23}^{N R^{*}} \\
+j \omega W_{12}^{R^{*}} & Z_{22} & +j \omega W_{23}^{R^{*}} \\
-W_{23}^{N R} W_{12}^{N R^{*}} & -j \omega W_{23}^{R} & Z_{33}
\end{array}\right]\left[\begin{array}{c}
\Gamma_{1} \\
\Gamma_{2}^{r} \\
\Gamma_{3}
\end{array}\right]=\left[\begin{array}{c}
Q_{1} \\
0 \\
0
\end{array}\right]
$$

This system gives a new interpretation of the modal interaction compared to the schema proposed in Fig. 3. The resonant modes of the structure remains connected to the resonant modes of each cavity by gyroscopic elements. The non resonant modes are no longer explicitly represented but their behaviours are simulated through direct couplings between the resonant modes of the two cavities. The coupling elements introduce coupling force proportional to the modal amplitudes (and not their time derivatives). They are then spring of stiffness given by the modal interaction interworks between the resonant cavity modes and the non resonant structure modes. An illustration of the new modal interaction scheme is proposed of Fig. 4. Compared to the former scheme (i.e. Fig. 3), it has the advantage to involve only couplings between resonant modes. The same process used for establishing the original SmEdA model can then be applied to the present problem. The energy formulation can be based on the power flow relation for two coupled oscillators excited by white noise source as emphasized in the next section.

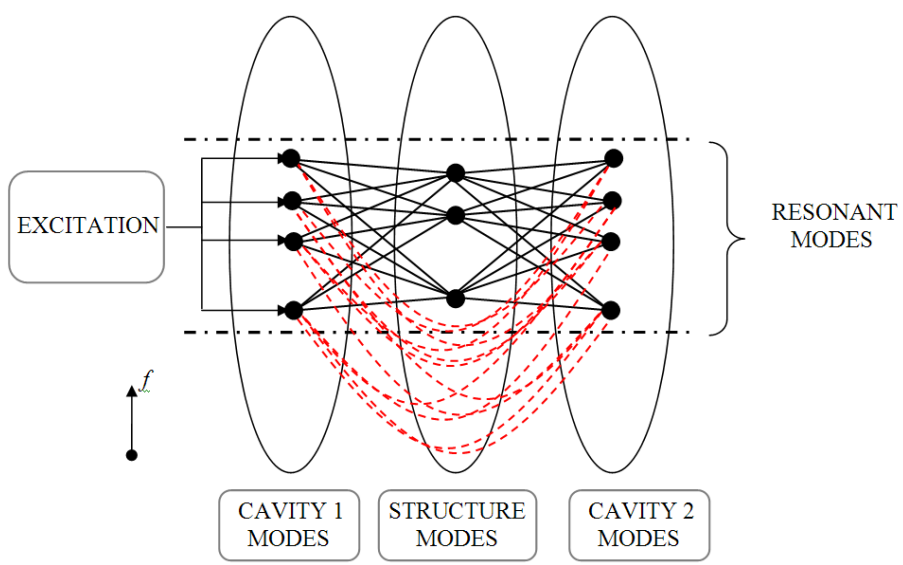

FIGURE 4. New interpretation of the modal interaction for the sound transmission problem below the critical frequency. Back, gyroscopic coupling; red, spring coupling. 


\section{Energy formulation}

SmEdA formulation is presented in details in [7]. One gives here the outline of this formulation.

First, it is based on the estimation of the energy sharing by two coupled modes from a relation established by Scharton and Lyon [11] considering two oscillators excited by white noise forces and coupled by a spring and a gyroscopic element.

The power flow exchanged by the mode $p$ of the cavity 1 and the mode $r$ of the cavity 2 which are coupled by a spring can be related to their modal energies by

$$
\Pi_{p r}=\beta_{p r}\left(E_{p}-E_{r}\right)
$$

where $\beta_{p r}$ is called the modal coupling factor. It is a function of natural angular frequencies, $\omega_{p}, \omega_{r} ;$ modal damping factors, $\eta_{p}, \eta_{r}$; and interaction modal works, $\mathrm{W}_{p q}, \mathrm{~W}_{r q}$ :

$$
\beta_{p r}=\left(\sum_{q \in Q_{n r}} W_{p q} W_{r q}\right)^{2}\left\{\frac{\left(\omega_{p} \eta_{p}+\omega_{r} \eta_{r}\right)}{\left[\left(\omega_{p}\right)^{2}-\left(\omega_{r}\right)^{2}\right]^{2}+\left(\omega_{p} \eta_{p}+\omega_{r} \eta_{r}\right)\left[\omega_{p} \eta_{p}\left(\omega_{r}\right)^{2}+\omega_{r} \eta_{r}\left(\omega_{p}\right)^{2}\right]}\right\} .
$$

The power flow exchanged by the mode $p$ of the cavity 1 and the mode $q$ of the structure which are coupled by a gyroscopic element can be evaluated by the same way.

Using these power flow relations and writing the power balance equation of each mode of the three subsystems, one obtains a linear equation system having the modal energy as unknowns:

$$
\begin{cases}\omega_{p} \eta_{p} E_{p}+\sum_{q \in \hat{Q}_{r}} \beta_{p q}\left(E_{p}-E_{q}\right)+\sum_{r \in \hat{R}} \beta_{p r}\left(E_{p}-E_{r}\right)=\Pi_{i n j}^{p}, & \forall p \in \hat{P}, \\ \omega_{q} \eta_{q} E_{q}+\sum_{p \in \hat{P}} \beta_{p q}\left(E_{q}-E_{p}\right)+\sum_{r \in \hat{R}} \beta_{q r}\left(E_{q}-E_{r}\right)=0, & \forall q \in \hat{Q}_{r}, \\ \omega_{r} \eta_{r} E_{r}+\sum_{q \in \hat{Q}_{r}} \beta_{q r}\left(E_{r}-E_{q}\right)+\sum_{p \in \hat{P}} \beta_{p r}\left(E_{r}-E_{p}\right)=0, & \forall r \in \hat{R},\end{cases}
$$

where $\Pi_{i n j}^{p}$ characterizes the acoustic source and corresponds to the modal injected power by the generalized source strength $Q_{p}$. This equation system can be solved and the total energy of each cavity can be finally obtained by adding modal energies:

$$
E_{C 1}=\sum_{p \in \hat{P}} E_{p}, \quad E_{C 2}=\sum_{r \in \hat{R}} E_{r},
$$

where $E_{C 1}$ (resp. $E_{C 2}$ ) is the time-averaged total energy of cavity 1 (resp. cavity 2). The classical Transmission Loss (TL) can then be easily deduced from these energies (see [1]).

\section{A BASIC EXAMPLE OF APPLICATION}

One illustrates here the present approach on an academic case for which the subsystem modes can be calculated analytically. One emphasizes that this approach could be easily applied to a complex thin structure coupled to cavities of complex geometries. In this case, the modal information (modal frequencies, modes shapes on the structure surface) can be evaluated by Finite Elements calculations for each uncoupled subsystem.

The considered case is shown on Fig. 5. It is composed of a rectangular simply-supported plate coupled on both side with a parallelepiped cavity. The plate has $0.5 \mathrm{~m} \mathrm{x} 0.4 \mathrm{~m}$ as dimension, $1 \mathrm{~mm}$ as thickness and is made of steel. The critical frequency of the plate is around $12 \mathrm{kHz}$. 


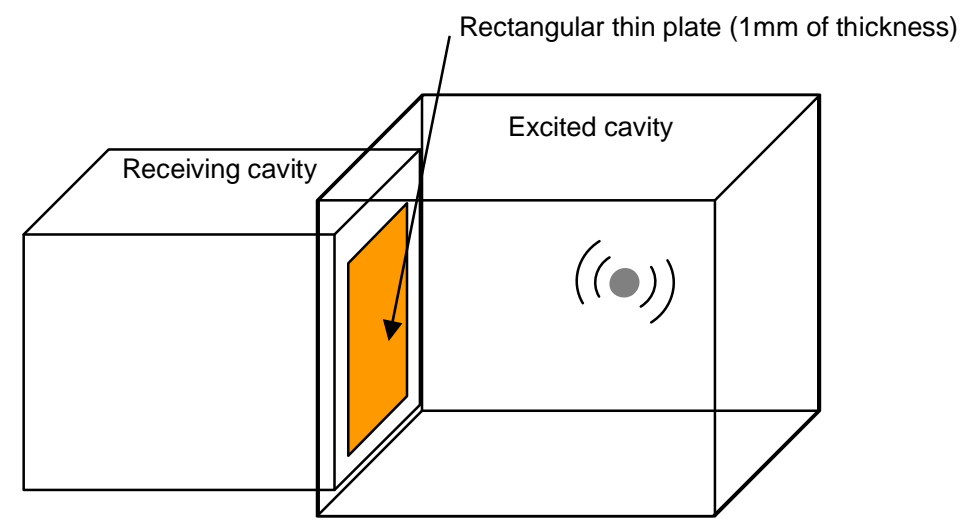

FIGURE 5. Illustration of the sound transmission between two parallelepiped cavities coupled by a rectangular plate.

For the third octave $1000 \mathrm{~Hz}$, Fig. 6 gives the values of the modal interaction works (see (4)) between the resonant cavity modes and the resonant/non resonant plate modes. The highest values are observed for the NR plate modes with a low modal order corresponding to modal frequencies below $200 \mathrm{~Hz}$. These modes are not in frequency coincidence with the resonant cavity modes but they are in space coincidence with the cavity modes (as highlighted by the values of Fig. 6).
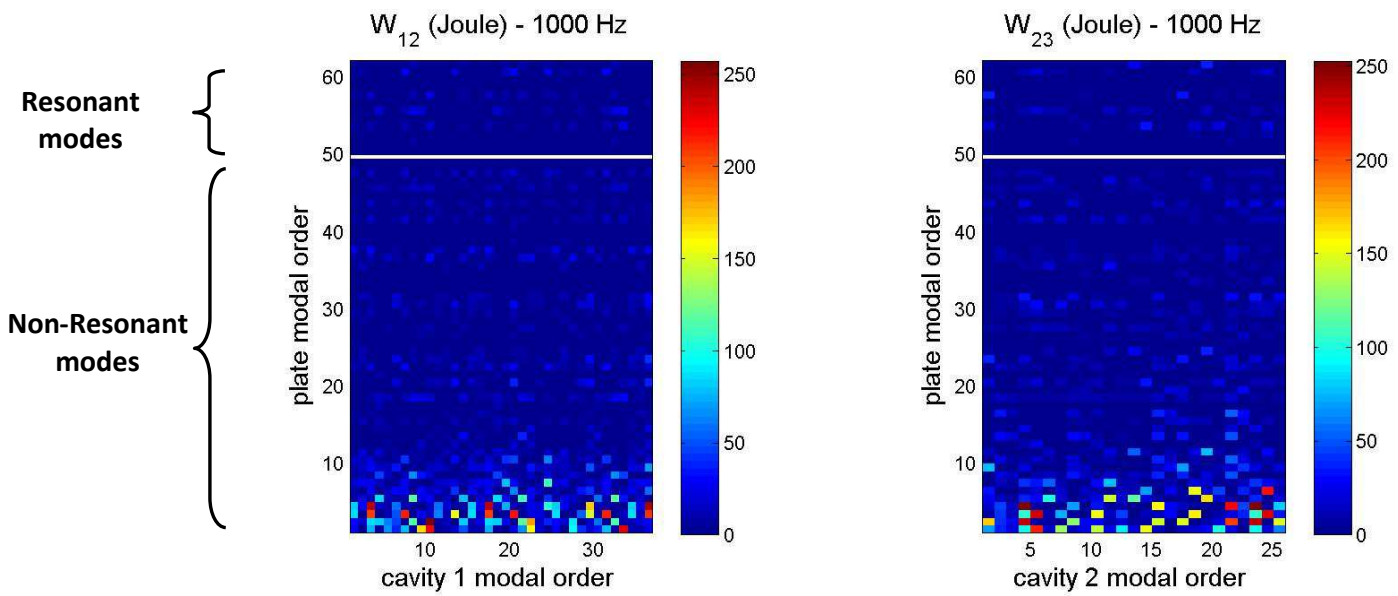

FIGURE 6. Interaction modal works: left, between the excited cavity and the plate; right, between the plate and the receiving cavity. Third octave band $1000 \mathrm{~Hz}$.
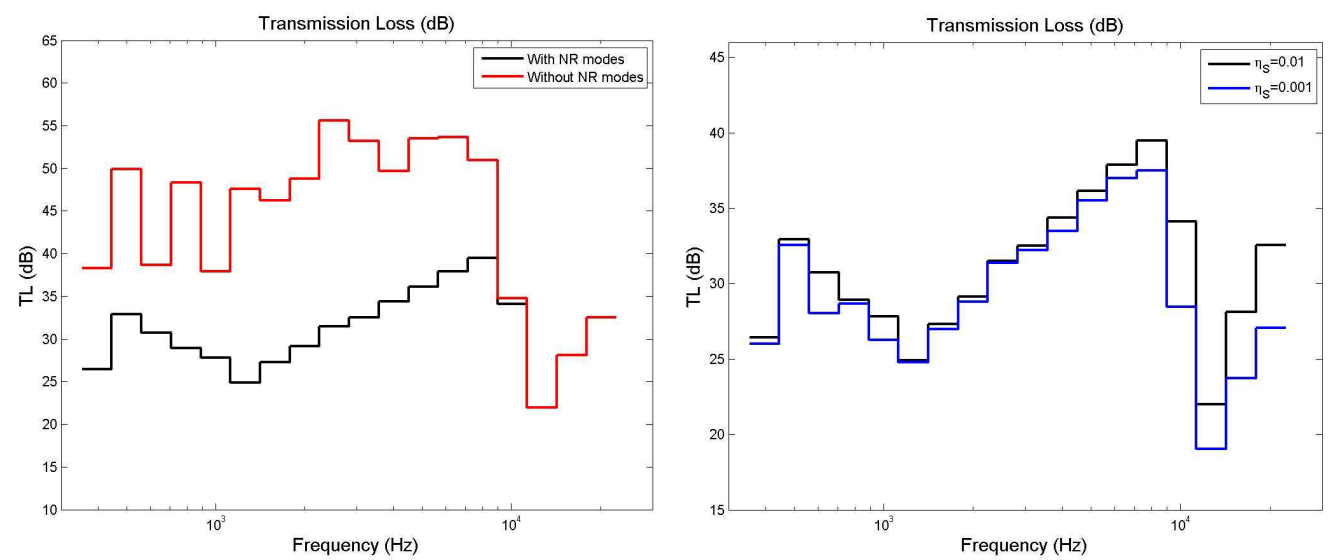

FIGURE 7. Transmission Loss: left, comparison of the SmEdA predictions with or without taking the NR modes into account; right, influence of the plate damping loss factor. 
Fig. 7 gives the Transmission Loss (TL) predicted by SmEdA. On the left, one compares the results of two calculations: one including the NR modes, the other taking only the resonant modes into account (i.e. original SmEdA). As it could be expected, one observes important discrepancies ( $>15 \mathrm{~dB}$ ) between the two calculations for the frequencies below the critical frequency. For frequencies between $1 \mathrm{kHz}$ and $10 \mathrm{kHz}$, the black curve corresponds to the mass law of the plate (i.e. slope of $6 \mathrm{~dB} /$ octave). On the right of Fig. 7, the SmEdA results including the NR modes are plotted for two different plate Damping Loss Factors (DLF): 0.01 and 0.001. Below the critical frequency, the DLF variation does not greatly influence the TL. This result could be expected because the damping has only an effect on the resonant contributions.

\section{PERSPECTIVES}

An extension of SmEdA taking the NR modes contributions into account has been presented. It will allow us using this model for predicting the TL of complex structures like a car firewall or a truck floor including the geometry of the engine and passenger compartments. The modal information (modal frequencies, modes shapes on the structure surface) can be evaluated by Finite Elements calculations for each uncoupled subsystem in a large frequency band (mid-frequency expected). Moreover, including a methodology developed recently in the laboratory [12], the SmEdA model could take into account the trim or damped material in the prediction.

\section{ACKNOWLEDGMENTS}

This work was co-funded by the French government (FUI 12 - Fonds Unique Interministériel) and European Union (FEDER - Fonds européen de développement régional ). It was carried out in the framework of the research project CLIC ("City Lightweight Innovative Cab") labelled by LUTB cluster (Lyon Urban Truck and Bus), in partnership with Renault Trucks, Arcelor-Mittal, ACOEM, CITI Technologies, FEMTO-ST (Univ. de Franche-Comté) and LVA (INSA de Lyon).

\section{REFERENCES}

[1] M. J. Crocker, A. J. Price. Sound transmission using statistical energy analysis. Journal of Sound and Vibration, 9469 486 (1969).

[2] A.J. Price, M.J. Crocker, Sound transmission through double panels using statistical energy analysis, Journal of the Acoustical Society of America, 47 683-693 (1970).

[3] R.J. Craik, L. Galbrun, Vibration transmission through a frame typical of timber-framed buildings, Journal of Sound and Vibration, 281 763-782 (2005).

[4] C. Churchill, C. Hopkins, L. Krajci, Modelling airborne sound transmission across a hybrid heavyweight-lightweight floor using statistical energy analysis. Proceeding of Forum Acusticum, Aalborg, Denmark, 2011.

[5] J-D, Chazot, J-L Guyader, Prediction of transmission loss of double panels with a patch mobility method, Journal of the Acoustical Society of America 121 267-278 (2006) .

[6] R. Craik, Non-resonant sound transmission through double walls using statistical energy analysis, Applied Acoustics, 64 325-341 (2003).

[7] L. Maxit, J.L. Guyader, Extension of SEA model to subsystem with non-uniform modal energy distribution, Journal of Sound and Vibration, 265 337-358 (2003) .

[8] L. Maxit, J.-L. Guyader, Estimation of SEA coupling loss factors using a dual formulation and FEM modal information. Part I: Theory, Part II: Numerical applications, Journal of Sound and Vibration, 239, 907-948 (2001).

[9] N. Totaro, C. Dodard, J.L. Guyader, SEA coupling loss factors of complex vibro-acoustic systems, Transaction of the ASME: Journal of Vibration and Acoustics, 131, 041009-1 (2009).

[10] F. Fahy, Vibration of containing structures by sound in the contained fluid. Journal of Sound and Vibration, 10 490-512 (1969).

[11] T.D. Scharton, R.H. Lyon, Power flow and energy sharing in random vibration. Journal of the Acoustical Society of America, 43 1332-1343 (1968).

[12] H. D. Hwang, K. Ege, L. Maxit, N. Totaro, J.-L. Guyader, A methodology for including the effect of a damping treatment in the mid-frequency domain using SmEdA method. Proceeding of ICSV20, Bangkok, Thailand, 7-11 July 2013. 